\title{
Improving biosensor analysis
}

\author{
David G. Myszka* \\ Huntsman Cancer Institute, University of Utah, Salt Lake City, UT 84132, USA
}

\begin{abstract}
The quality of optical biosensor data must be improved in order to characterize the mechanism and rate constants associated with molecular interactions. Many of the artifacts associated with binding data can be minimized or eliminated by designing the experiment properly, collecting data under optimum conditions and processing the data with reference surfaces. It is possible to globally fit high-quality biosensor data with simple bimolecular reaction models, which validates the technology as a biophysical tool for interaction analysis. Copyright (C) 1999 John Wiley \& Sons, Ltd.
\end{abstract}

Keywords: BIACORE, surface plasmon resonance; kinetics; protein-protein interactions; biosensor

Received 2 July 1999; accepted 5 July 1999

\section{Introduction}

While commercial biosensors (BIACORE and IAsys) are simple to operate, accurately interpreting binding reactions is not always straightforward. Since the majority of published biosensor data do not fit a simple bimolecular interaction model $(\mathrm{A}+\mathrm{B}=\mathrm{AB})$, many investigators are concerned about the validity of biosensor analysis. However, the inability to fit data to a simple model is often a result of how the experiments are run and not a flaw in the technology. Many investigators collect data under conditions that are not suitable for measuring binding kinetics. There are a number of experimental artifacts that can complicate biosensor analysis, including surface-imposed heterogeneity, mass transport, aggregation, avidity, crowding, matrix effects and nonspecific binding (Myszka, 1997; Morton and Myszka, 1998). Improving the design of biosensor experiments, as well as improving the way binding data are collected and processed, can eliminate most of these artifacts (Myszka, 2000). By improving the quality of the sensor data, we have described a number of systems with simple interaction models (Myszka et al., 1996; Roden and Myszka, 1996; Myszka et al., 1997; Stuart et al., 1998). For example, Fig. 1 shows a data set for an IL2-receptor interaction globally fit with a simple interaction model (Myszka, 2000). Note that the association and dissociation phases responses for each IL-2 concentration are described very well by this simple model. This article highlights the key steps required to improve the quality of data when the goal is to interpret the binding kinetics recorded on biosensors.

\footnotetext{
* Correspondence to: D. G. Myszka, Huntsman Cancer Institute, University of Utah, 50 N. Medical Drive, School of Medicine no. 4A417, Salt Lake City, UT 84132, USA. E-mail: dmyszka@hci.utah.edu
}

Abbreviations used: IFC, integrated fluidic cartridge RU, resonance units.

\section{Step 1. Improving the experimental design: Generating high quality biosensor data takes work}

\section{Start with good reagents}

The quality of biosensor data is directly proportional to the quality of the reagents. For a detailed biophysical study, both analyte and ligand should be chemically and conformationally pure. In order for data to fit a simple bimolecular reaction model, the analyte and ligand most be monomeric in solution and form a 1:1 complex when mixed. Investigators often assume but rarely demonstrate that their

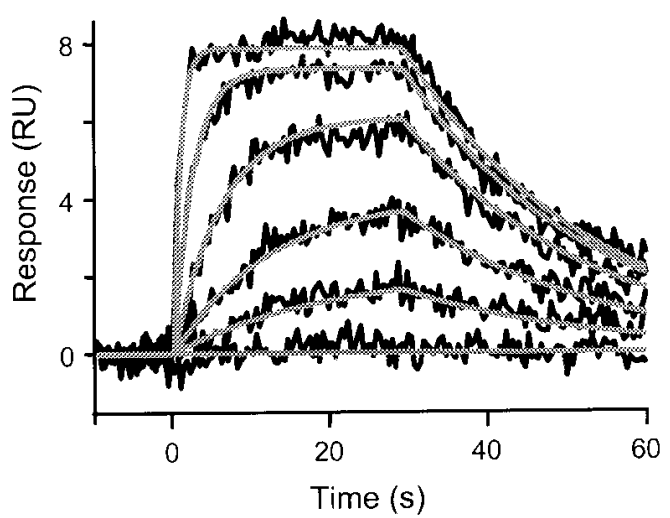

Figure 1. Global analysis of biosensor data. Black lines are an average of four repeat injections of IL-2 over an IL-2 $\alpha$-receptor surface (see Myszka, 2000). The IL-2 concentrations were 233, 78, $26,8.6,2.9$ and $0 \mathrm{nM}$. The running buffer contained $10 \mathrm{~mm}$ sodium phosphate, $\mathrm{pH} 7.4,150 \mathrm{mM}$ sodium chloride, $0.005 \% \mathrm{p} 20$, and $0.1 \mathrm{mg} / \mathrm{ml}$ bovine serum albumin. Binding data were collected at a flow rate of $100 \mu \mathrm{l} / \mathrm{min}$. Grey lines represent a best fit of the binding data to a simple bimolecular reaction model $(A+B=A B)$. The association and dissociation phase data for each concentration of IL-2 were fit simultaneously using CLAMP (Myszka and Morton, 1998; Morton and Myszka, 1998). 
reagents are well behaved. Analytical ultracentrifugation can be used to determine the assembly state of macromolecules in solution and is an excellent corroborative tool for biosensor analysis (Hensley, 1996). In general the biosensor should be viewed as a biophysical instrument and, as with any high-resolution technique, the better the reagents the better the results.

\section{Instrument cleaning}

Generating high-quality data demands that the instrument be in good condition. Once a week we run a series of washing steps to keep the BIACORE fluidic system and syringe pumps clean and in good working order. This includes priming the system four times in a row with each of the following reagents: $0.5 \%$ SDS, $6 \mathrm{M}$ urea, $1 \%$ acetic acid and $0.2 \mathrm{M} \mathrm{NaHCO}_{3}$. If the instrument is not going to be used for a period of $12 \mathrm{~h}$ or more, we prime the system with water and run a method that injects an aliquot of water every $30 \mathrm{~min}$. This helps reduce salt build-up in the flow system. Whenever the running buffer is changed, it is important to prime the system at least three times before beginning any experiment.

\section{Check baseline}

To ensure that the instrument is operating properly, a sample of the same running buffer should be injected at the start of the experiment. Ideally one would expect to see a completely flat response, but often there will be a positive bulk refractive index change. Usually this is minor (5-10 RU) and consistent between the four flow cells available on BIACORE 2000 and 3000 instruments. If the responses on the different flows are not the same, it may be an indication that the IFC (integrated fluidic cartridge) needs replacing.

Finally, it is important to ensure that the baseline response does not drift. Baseline drift is usually caused by temperature fluctuations, which will decrease as the instrument equilibrates at the beginning of an experiment. Drift in excess of an RU per minute, or drift that is not consistent between flow cells, may be an indication that the IFC or sensor chip needs replacing.

\section{Check for nonspecific binding}

Biological macromolecules often show a proclivity to interact with surfaces. It is necessary to check both reagents for nonspecific binding on the biosensor before running any experiments. The best way to do this is to simply inject each sample over a nonderivatized surface at the highest concentration to be used in the analysis. A low level of background binding may be compensated for using a reference surface, which will be discussed below. If the nonspecific binding is high, then the experimental conditions may be altered. For example, basic proteins tend to bind to the highly charged carboxymethyl dextran matrix. In these cases, we have found that the Pioneer chip B1, which has a lower charge density, dramatically reduces nonspecific binding. If the nonspecific binding cannot be eliminated, it

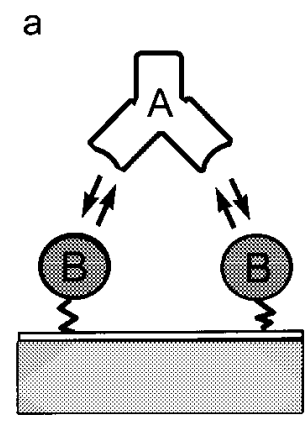

b

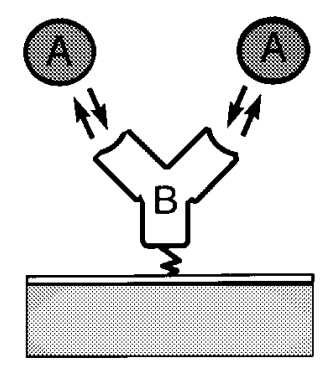

Figure 2. Assay orientation. (a) The binding of a bivalent analyte (A) to monovalent ligand (B) immobilized on the biosensor chip. (b) The binding of monovalent analyte to the bivalent ligand immobilized on the surface. The analyte in (a) has the potential to cross-link on the surface and generate avidity effects, which is avoided with the orientation shown in (b).

is best to couple the sticky protein onto the surface. Be cautious though, as the nonspecific binding may affect the activity of the immobilized ligand.

\section{Avoid avidity effects}

If the goal is to describe binding data with a simple interaction model, then the assay must be designed properly. For example, bivalent molecules such as antibodies should always be immobilized onto the surface to avoid avidity effects. If an antibody is in solution, it has the potential to cross-link with two antigens on the surface, as illustrated in Fig. 2. This will result in an apparent higher affinity and the kinetics cannot be described with a simple interaction model. In some cases the ability to cross-link molecules on the dextran surface can be an advantage. We have studied the interaction of IL-2 ligand with different receptor subunits immobilized on the same surface (Myszka et al., 1996). The flexibility of the dextran matrix and high local concentrations of immobilized receptors made it possible to form complexes that mimic the cell surface better than solution phase assays.

\section{Use oriented immobilization}

While biosensors do not require reactants to be labeled, one of the molecules must be immobilized onto a surface. As shown in Fig. 3, direct coupling and capturing are the two general ways of immobilizing the ligand. Direct coupling [Fig. 3(a)] has the advantage of creating a stable surface. 

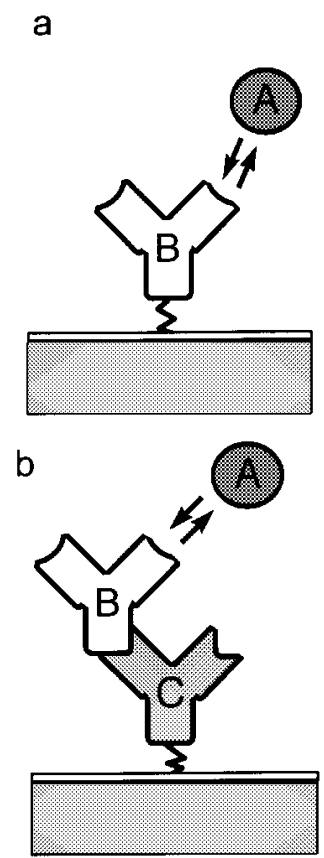

Figure 3. Immobilization methods. (a) The direct coupling of the ligand (B) onto the biosensor chip. Commonly used coupling chemistries utilize amines, aldehydes or free thiols on the surface of the ligand (O'Shannessy et al., 1992). Direct coupling produces a stable surface, but it may introduce surface heterogeneity. (b) An example of a capturing system. Here the ligand (B) is first captured onto the surface using another immobilized molecule (C). Common capturing methods include $6 \times$ His, FLAG or GST fusion systems, as well as antibodies, protein A or G and biotin/ streptavidin. Capturing produces a homogeneous surface, but it may not be stable.

Coupling through unique surface-exposed residues such as an engineered cysteine is optimal (Myszka et al., 1996). Random coupling procedures, such as chemistry involving primary amines, often introduces surface heterogeneity, which will complicate the analysis. Capturing methods [see Fig. 3(b)], such as antibodies or fusion tags, create homogeneous surfaces, but if the capturing step is not stable it may introduce a background surface decay which also complicates the analysis (Joss et al., 1998). Selecting the optimum immobilization method is entirely dependent on the system and often involves a compromise between introducing surface heterogeneity or surface instability. In the end, the hard work that goes into finding an optimum immobilization procedure will pay off by generating binding responses that are easy to analyze.

\section{Step 2. Improve data collection: consider all variables in collecting biosensor data}

\section{Reference surfaces}

A reference surface can dramatically improve the quality of the binding data by correcting for artifacts such as bulk refractive index changes, matrix effects, nonspecific binding, injection noise and baseline drift (Morton and Myszka, 1998; Myszka, 2000). It is best to treat the reference surface

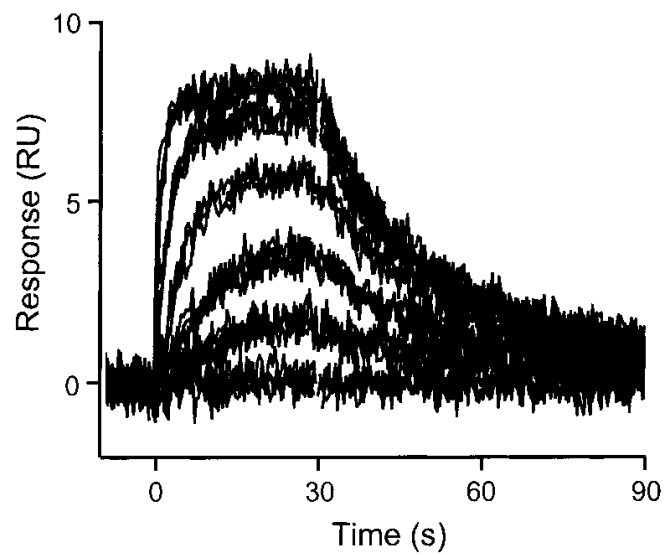

Figure 4. Checking the reproducibility of binding responses. The same concentrations of IL-2 $(233,78,26,8.6,2.9$ and $0 \mathrm{nM})$ were injected over an $\alpha$ RIL-2 surface four times each. The figure shows an overlay of the corrected binding responses.

with the same immobilization conditions used for the reaction surface to ensure the environment between the flow cells is similar. When measuring the interactions of small molecular weight analytes, it is particularly important to immobilize a noninteracting protein at the same density. This helps to normalize the refractive index changes between sensor surfaces. The benefits of using a reference surface will be illustrated in the Data Analysis section below. BIACORE 2000 and 3000 instruments can measure reactions for the same analyte over four different surfaces simultaneously. This makes setting up and collecting data from reference surfaces convenient. It is still beneficial to use reference surfaces in cases where instruments do not allow simultaneous analysis, however, it is more demanding.

\section{Surface capacity}

When performing kinetic experiments the capacity of the sensor surface should be kept low to minimize mass transport, steric hindrance, crowding and aggregation. With BIACORE 2000 and 3000 it is relatively simple to measure binding responses with a maximum response less than 50 RU. In fact, reproducible data can be collected below 10 RU, as shown in Fig. 4. In this example, each IL-2 injection was repeated four times and the responses are all overlaid. While these data contain a high level of random noise, the binding responses themselves are reproducible. We have demonstrated that, when using global analysis, the random noise has little effect on the accuracy of the parameter estimates (Myszka et al., 1998). By going to very low capacity surfaces many artifacts can be avoided that would otherwise complicate the analysis.

\section{Flow rate and sample plug}

High flow rates are important for minimizing mass transport effects (Myszka, 1997; Myszka et al., 1998). Figure 5 shows an example of varying the flow rate to test whether a reaction is limited by mass transport (Morton and Myszka, 


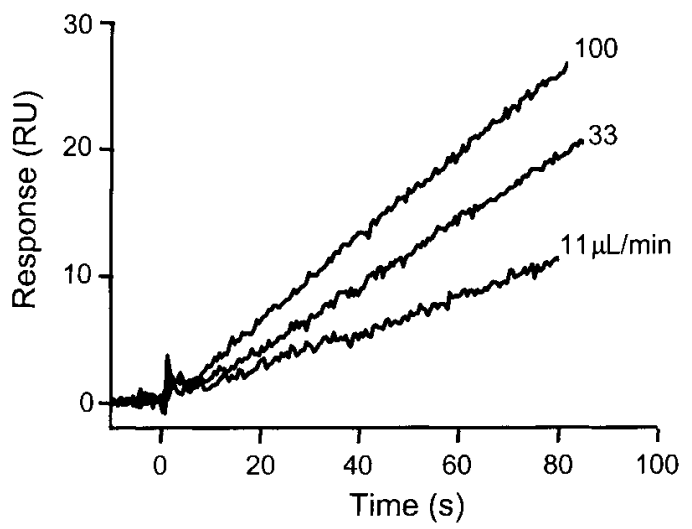

Figure 5. Checking for flow rate dependence. The same concentration of erythropoietin ligand $(0.66 \mathrm{nM})$ was injected over an erythropoietin receptor surface at flow rates of 100, 33 and $11 \mu \mathrm{l} / \mathrm{min}$ as labeled (Morton and Myszka, 1998). The increase in binding rate with increasing flow rate is an indication that this reaction is affected by mass transport (Myszka et al., 1998).

1998). Indeed in this case the binding rate increased with increasing flow rate. For this system, kinetic data were collected at the highest flow rate possible to minimize transport effects. High flow rates also help to deliver a consistent sample plug, because of faster switching between running buffer and sample.

It is also important to limit the volume of the injected sample to avoid dispersion of the sample plug. If during long injections the binding responses reach equilibrium and begin to decrease before the end of the association phase, the injection volume needs to be lowered. Nonuniform sample plugs may be an indication that the system needs cleaning or that the IFC needs replacing. One method of testing whether the sample plug is uniform is to inject running buffer with some added salt.

\section{Washing steps}

In BIACORE instruments the same microfludic system and needle are used to deliver samples and regeneration buffer to the sensor surface. The analysis can be complicated by small amounts of regeneration buffer carried over between reaction cycles. To eliminate this concern, it is best to run a series of blank injections and washing steps for each cycle, as shown in Fig. 6. A blank injection before the sample ensures the injection needle, tubing and flow-path are clean. Each regeneration step is followed by an IFC wash procedure as well as another blank injection. If the regeneration reagent gives a large refractive index change, a small change will be noticed in the following injection, even after the washing step. Without these careful washing procedures, some of the regeneration buffer will contaminate the sample plug and affect the next binding reaction.

\section{Replicate and randomize samples}

In order to perform a statistical analysis of the reaction data, each analyte concentration should be repeated and the order of injection randomized. Sample replication is an under-

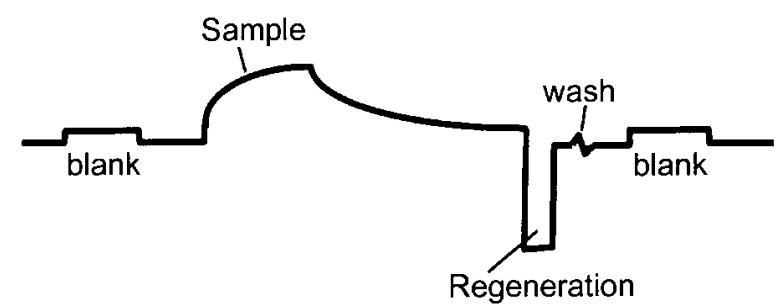

Figure 6. Injection cycles. The flow system needs to be washed between binding cycles in order to minimize sample carry over. The IFC wash and blank injections prevent regeneration buffer from contaminating the following sample.

lying requirement for any scientific experiment, but unfortunately it is often neglected in biosensor analysis. Replicate experiments provide a model-independent assessment of the total experimental noise, taking into account changes in analyte or ligand activity over time and the efficiency of the regeneration step. Randomizing the samples is essential for removing any bias in the data related to when the experiments were carried out.

Because most BIACORE instruments are automated, replicate injections should be standard practice in these systems. It is also more beneficial to replicate a small number of samples over a wide concentration range as opposed to performing single injections of a large number of concentrations. Typically analyte concentrations are varied 100 -fold and each injection replicated three to five times. It is also imperative to run a blank injection along with the samples, for reasons discussed below.

\section{Step 3. Improve data processing: biosensor data must be processed in order to remove system artifacts}

Figure 7(a) shows raw data collected for IL-2 ligand injected over a surface containing IL- $2 \alpha$-receptor as well as a blank reference surface (Myszka, 2000). The capacity of the receptor surface was kept low to avoid mass transport effects. It is perhaps difficult to discern the parts of the response that are a result of the IL-2-receptor interaction over all of the other system artifacts. Indeed the noise from the injection needle, bulk refractive index change and washing steps are similar in magnitude to the reaction data. All of these artifacts can be removed by processing the data.

\section{Zeroing}

The first step in data processing is to zero the response on the $y$-axis just prior to the start of the association phase. Normally it is best to subtract an average of the response a few seconds before the injection, which results in the plot shown in Fig. 7(b). The start of the injection on the time axis ( $x$-axis) can be set to zero by eye. When collecting data from multiple surfaces it is important to set the injection time to zero separately for each flow cell. With the expanded scale in Fig. 7(b), it is easier to see the differences between the reaction and reference surface data, as well as their similarities. Many of the injection artifacts are identical 

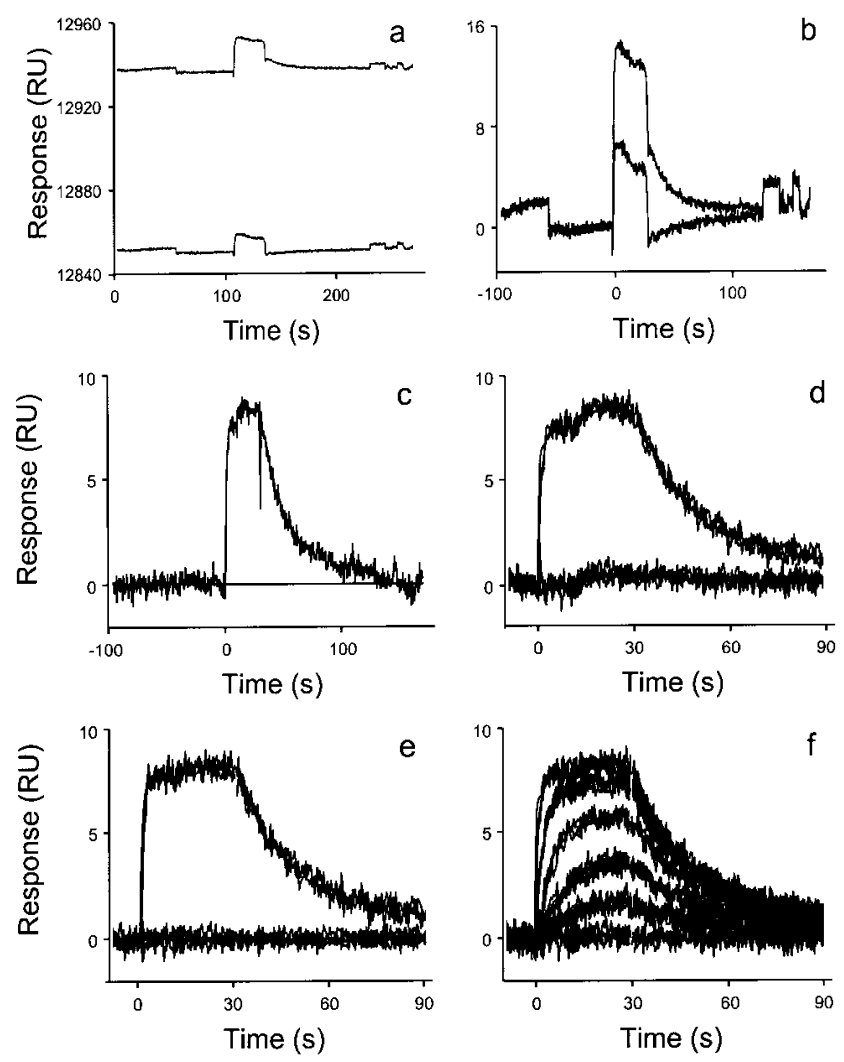

Figure 7. Processing biosensor data. (a) Raw data from biosensor for 233 nM IL-2 injected over a receptor surface (top trace) and reference surface (bottom trace). (b) Data sets were zeroed on the $y$ and $x$ axis just prior to the start of the injection. (c) Data from the reference surface was subtracted from the data from the reaction surface. (d) Overlay of four replicate injections of $233 \mathrm{nM}$ IL-2 as well as a running buffer blank. (e) After subtracting the average of the blank injections from both the sample and blank data. (f) Overlay of a series of IL-2 injections $(233,78,26,8.6,2.9$ and $0 \mathrm{nM}$ ) replicated four times each.

between these surfaces. For example, there is a small downward drift during the association phase which occurs in the reaction and reference flow cells.

\section{Reference subtraction}

Subtracting the reference surface data from the reaction surface data eliminates the majority of the refractive index change and injection noise, as shown in Fig. 7(c). It is easier to see the binding response attributed to the IL-2-receptor reaction. The differences in the position of the reaction and reference flow cells in the IFC cause sharp spikes at either the beginning or end of the association phase. These outlier data points should be eliminated during data analysis in order to get a proper representation of the quality of the fit.

\section{Replication overlays}

In Fig. 7(d), the reference subtracted responses have been overlaid for four replicate injections of the same IL-2 concentration as well as a set of blank injections. At the end of the section on Improving Data Collection, the point was made to run blank injections along with the analyte samples. Looking closely at the blank injections a small systematic drift is noticeable in the data about $10 \mathrm{~s}$ into the association phase. The signal drifts up about 1 RU and then it stabilizes. Note that the exact same drift is observed in the analyte responses. While the magnitude of the drift is small, at these low surface densities it makes a significant contribution to the binding response. These minor systematic deviations are often seen in the binding response when working on lowcapacity surfaces and they are specific for each flow cell. The next section describes how to correct for these effects.

\section{Double referencing}

The systematic deviations seen in responses measured on low-capacity surfaces is extremely reproducible and occurs equally in all the analyte injections as well as the blank. Therefore, the response from the blank injections can be used to remove this artifact from all of the data in a procedure referred to as 'double referencing'. The plots shown in Fig. 7(e) were generated by subtracting the average of the response of the blank injections from the entire data set. Notice that the baseline data are completely flat and the responses from the sample data are stable once they reach equilibrium. This procedure dramatically improves the quality of data, making it possible to collect reliable data on BIAcore 2000 below 2 RU. Double referencing will be particularly useful for collecting data on low molecular weight analytes, since the binding responses are small to begin with.

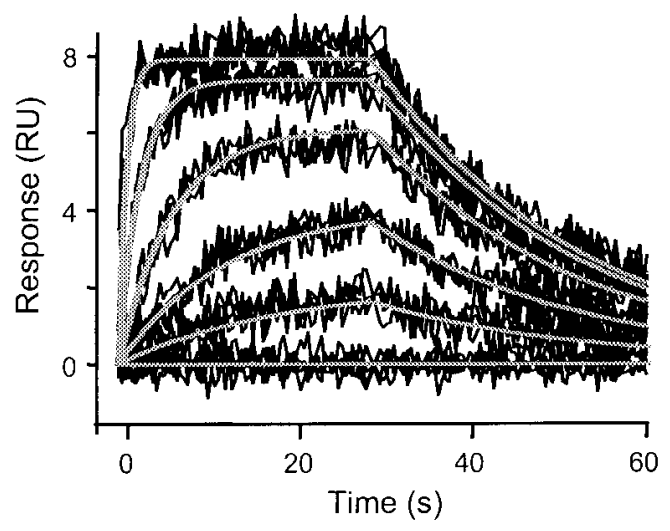

Figure 8. Global fit of the IL-2 ligand-receptor interaction data. Black lines constitute four repeat injections of IL-2 over an IL-2 $\alpha$ receptor surface. The IL-2 concentrations were $233,78,26,8.6,2.9$ and $0 \mathrm{nM}$. More details on the experimental conditions may be found in Myszka (2000). Grey lines represent a best fit of the binding data to a simple bimolecular reaction model $(A+B=A B$, Myszka and Morton, 1998). The residual standard deviation was $0.409 \mathrm{RU}$, which is less than $0.05 \mathrm{RU}$ greater than the replication standard deviation of $0.365 \mathrm{RU}$. The replication standard deviation is a model independent assessment of the total experimental noise (Morton and Myszka, 1998). The small difference between the residual and replication standard deviation indicates that there is very little information in the data that is not accounted for by the reaction model. The returned association $\left(k_{\mathrm{a}}\right)$ and dissociation rate $\left(k_{\mathrm{d}}\right)$ constants were $1.01(1) \times 10^{7} / \mathrm{M} / \mathrm{s}$ and $0.213(3) / s$, respectively. The numbers in parentheses represent the standard error in the last significant digit. A fit to the average of the experimental data is shown in Fig. 1. 


\section{Overlay all analyte responses}

The final step in preparing the sensor data for analysis is to overlay the response from the different analyte concentrations, as shown in Fig. 7(f). These responses represent an example of high-quality biosensor data. Responses were collected over a wide analyte concentration range. The binding capacity was kept low to avoid mass transport effects. Replicate injections demonstrate that the responses are reproducible and the responses come to equilibrium and dissociate back to baseline.

\section{Globally fitting data}

Once the quality of the biosensor data has been improved, interpreting the binding kinetics is easy. The IL-2-receptor interaction data were globally fitted (Morton and Myszka, 1998) to a simple interaction model $(\mathrm{A}+\mathrm{B}=\mathrm{AB})$ as shown in Fig. 8. This model, which requires only three parameters, an association rate constant $\left(k_{\mathrm{a}}\right)$, a dissociation rate constant $\left(k_{\mathrm{d}}\right)$ and a maximum surface capacity $\left(B_{\max }\right)$, describes the data for all of the analyte concentrations very well. Our global analysis software has been described previously (Myszka and Morton, 1998; Morton and Myszka, 1998) and is available at www.hci.utah.edu/groups/interaction. We were able to globally fit data collected on the IL-2-receptor system at four different temperatures, from 4 to $40{ }^{\circ} \mathrm{C}$ (see Myszka, 2000). The ability to globally fit data to simple interaction models from this system as well as several other binary protein systems (Roden and Myszka, 1996; Stuart et al., 1998) validates BIAcore as a tool for kinetic analysis of molecular interactions. We have also used global analysis to describe several reactions that were limited by mass transport (Myszka et al., 1996, 1997; Morton and Myszka, 1998). More information on the benefits of global analysis can be found in Morton et al. (1995), Myszka et al. (1997) and Morton and Myszka (1998).

\section{Summary}

Optical biosensors have the potential to provide detailed information about binding reactions. However, there are a number of artifacts that investigators must consider when designing biosensor experiments. Collecting data under the appropriate conditions and processing the data through the use of reference surfaces and blank injections can eliminate many system dependent artifacts. It is possible to describe high-quality biosensor data with simple interaction models, validating the technology as a biophysical tool for interaction analysis.

\section{Acknowledgements}

I would like to thank Thomas Ciardelli for providing the IL-2 ligand and receptor used in this report. This work was supported by the Huntsman Cancer Institute and Grant 2 P30 CA42014-12 from the National Cancer Institute.

\section{References}

Hensley, P. (1996) Defining the structure and stability of macromolecular assemblies in solution: the reemergence of analytical ultracentrifugation as a practical tool, Curr. Biol. 4: 367-373.

Joss, L. A., Morton, T. A., Doyle, M. L. and Myszka, D. G. (1998) Interpreting kinetic rate constants from optical biosensor data recorded on a decaying surface, Anal. Biochem. 261: 203-210.

Morton, T. A. and Myszka, D. G. (1998) Kinetic analysis of macromolecular interactions using surface plasmon resonance biosensors, Meth. Enzymol. 295: 368-294.

Morton, T. A., Myszka, D. G. and Chaiken I. M. (1995) Interpreting complex binding kinetics from optical biosensors: a comparison of linear analysis, the integrated rate equation and numerical integration. Anal. Biochem. 227: 176-185.

Myszka D. G. (1997) Kinetic analysis of macromolecular interactions using surface plasmon resonance biosensors. Curr. Opin. Biotechnol. 8: 50-57.

Myszka D. G. (2000) Kinetic, equilibrium and thermodynamic analysis of macromolecular interactions on BIACORE, Meth. Enzymol. in press

Myszka, D. G. and Morton, T. A. (1998) CLAMP: a biosensor kinetic data analysis program, TIBS 23, 149-150.

Myszka, D. G., Arulanantham, P. G., Sana, T., Wu, Z., Morton, T. A. and Ciardelli, T. L. (1996) Kinetic analysis of ligand binding to Interleukin-2 receptor complexes created on an optical biosensor surface, Protein Sci. 5: 2468-2478.

Myszka, D. G., Morton, T. A., Doyle, M. L. and Chaiken, I. M. (1997) Kinetic analysis of a protein antigen-antibody interaction limited by mass transport on an optical biosensor, Biophys. Chem. 64: 127-137.

Myszka, D. G., Xiaoyi, H., Dembo, M., Morton, T. A. and Goldstein, B. (1998) Extending the range of rate constants available from BIACORE: interpreting mass transport influenced binding data, Biophys. J. 75: 583-594.

O'Shannessy, D. J., Brigham-Burke, M. and Peck, K. (1992) Immobilization chemistries suitable for use in the BIAcore surface plasmon resonance detector, Anal. Biochem. 205, 132-136.

Roden, L. D. and Myszka, D. G. (1996) Global analysis of a macromolecular interaction measured on BIAcore, Biochem. Biophys. Res. Commun. 225: 1073-1077.

Stuart, J. K., Myszka, D. G., Joss, L., Mitchell, R. S., McDonald, S. M., Xie, Z., Takayama, S., Reed, J. C. and Ely, K. R. (1998) Characterization of interactions between the anti-apoptotic protein BAG-1 and Hsc 70 molecular chaperones, J. Biol. Chem. 273: 22506-22514. 